July 16, 2018 15:27 WSPC/INSTRUCTION FILE Brevik-casimirfrictionbenasque 2011

International Journal of Modern Physics: Conference Series

(C) World Scientific Publishing Company

\title{
CASIMIR FRICTION FORCE FOR MOVING HARMONIC OSCILLATORS
}

\author{
JOHAN S. HØYE \\ Department of Physics, Norwegian University of Science and Technology, N-7491 Trondheim, \\ Norway \\ johan.hoye@ntnu.no \\ IVER BREVIK \\ Department of Energy and Process Engineering, Norwegian University of Science and \\ Technology, N-7491 Trondheim, Norway \\ iver.h.brevik@ntnu.no \\ Received Day Month Year \\ Revised Day Month Year
}

\begin{abstract}
Casimir friction is analyzed for a pair of dielectric particles in relative motion. We first adopt a microscopic model for harmonically oscillating particles at finite temperature $T$ moving non-relativistically with constant velocity. We use a statistical-mechanical description where time-dependent correlations are involved. This description is physical and direct, and, in spite of its simplicity, is able to elucidate the essentials of the problem. This treatment elaborates upon, and extends, an earlier theory of ours back in 1992. The energy change $\Delta E$ turns out to be finite in general, corresponding to a finite friction force. In the limit of zero temperature the formalism yields, however, $\Delta E \rightarrow 0$, this being due to our assumption about constant velocity, meaning slowly varying coupling. For couplings varying more rapidly, there will also be a finite friction force at $T=0$. As second part of our work, we consider the friction problem using time-dependent perturbation theory. The dissipation, basically a second order effect, is obtainable with the use of first order theory, the reason being the absence of cross terms due to uncorrelated phases of eigenstates. The third part of the present paper is to demonstrate explicitly the equivalence of our results with those recently obtained by Barton (2010); this being not a trivial task since the formal results are seemingly quite different from each other.
\end{abstract}

Keywords: Casimir friction; Casimir effect.

PACS numbers: 05.40.-a, 05.20.-y, 34.20.Gj

\section{Introduction and background}

Consider the arrangement sketched in Fig. 1, namely two parallel semi-infinite dielectric nonmagnetic plates at micron or semi-micron separation, the upper plate kept at rest, the lower moving with a constant nonrelativistic velocity $V$. For simplicity we assume the media to be of equal composition, their permittivity being real, equal to $\varepsilon$. In principle, we may allow for dispersion. The electromagnetic force 

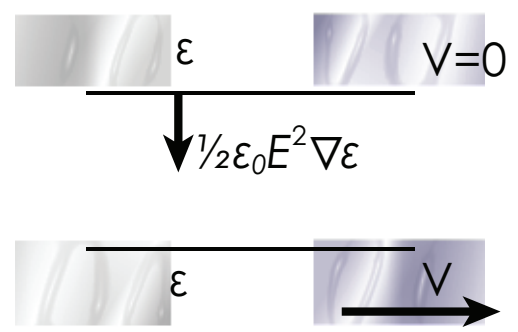

density in the boundary layer of the resting plate is

$$
\mathbf{f}=-\frac{1}{2} \varepsilon_{0} E^{2} \nabla \varepsilon
$$

The essential property of the force (11) in our present context is that it is vertically directed, i.e. transverse to the relative motion of the plates. And it is precisely this force which is able to explain all the conventional experiments in classical electrodynamics where dielectric media, especially liquids, are involved. Let us for illustration mention three examples:

- The first is the classic radiation pressure experiment of Ashkin and Dziedzic 1. Focused light was sent from above towards an air-water surface, and an outward bulge of the surface was observed, of the order of $1 \mu \mathrm{m}$. The light source was a pulsed frequency doubled YAG laser pulse (duration of each pulse $60 \mathrm{~ns}$, peak power $3 \mathrm{~kW}$, beam waist $4.5 \mu \mathrm{m}$ ). The reason for the smallness elevation is the large surface tension for air-water. Theoretical papers on this experiment can be found in Refs. 2 and 3 .

- Our second example is the experimental work of Zhang and Chang 4 , showing clearly how a water droplet becomes deformed when hit by a laser pulse. Typical laser energies were $100 \mathrm{~mJ}$ in each pulse. Theoretical treatments for this case are given in Refs. 5 and 6 .

- A newer series of experiments is provided by the Bordeaux group $7|8| 9$, working with a two-fluid system of surfactant-coated nanodroplets in oil microemulsions near the critical point, implying that the surface tension can be made about $10^{6}$ times smaller than the usual air-water tension. The surface displacement can accordingly be very large, about $70 \mu \mathrm{m}$.

In our context, the point to be emphasized is that in all these cases the expression (11) provides the correct electromagnetic force. And this brings us to the present theme: how can the Casimir friction force be accounted for, by the use of conventional electrodynamics? Obviously, the transverse force (11) cannot be of importance for friction. It is clear that we here have to leave the assumption of a real 
permittivity. Dissipation has to be rooted in energy dissipation, that means, in a permittivity that is complex valued.

It is worthwhile to show how one can come to the same conclusion via an alternative approach, namely with the use of field theory. Let us start from Schwinger's source theory, giving how the electric field components $E_{i}$ are related to the polarization source $P_{k}$ via a generalized susceptibility $\Gamma_{i k} 10$

$$
E_{i}(x)=\int d^{4} x \Gamma_{i k}\left(x, x^{\prime}\right) P_{k}\left(x^{\prime}\right) .
$$

Stationarity means that we can take the difference $\tau=t-t^{\prime}$ as time variable. Causality means that $t^{\prime} \leq t$. We take the Fourier transform to obtain the spectral representation $\Gamma_{i k}\left(\mathbf{r}, \mathbf{r}^{\prime}, \omega\right)$. The Kubo formula relates $\Gamma$ to the commutator:

$$
\Gamma_{i k}\left(\mathbf{r}, \mathbf{r}^{\prime}, \omega\right)=i \int_{0}^{\infty} d \tau e^{i \omega \tau}\left\langle\left[E_{i}(x), E_{k}\left(x^{\prime}\right)\right]\right\rangle .
$$

The generalized susceptibility is the same as the retarded Green function: $\Gamma_{i k}\left(x, x^{\prime}\right)=G_{i k}^{R}\left(x, x^{\prime}\right)$. Now take the Fourier transform of the two-point function $\left\langle E_{i}(x) E_{k}\left(x^{\prime}\right)\right\rangle$ :

$$
\left\langle E_{i}(\mathbf{r}, \omega) E_{k}\left(\mathbf{r}^{\prime}, \omega^{\prime}\right)\right\rangle=2 \pi\left\langle E_{i}(\mathbf{r}) E_{k}\left(\mathbf{r}^{\prime}\right)\right\rangle_{\omega} \delta\left(\omega+\omega^{\prime}\right),
$$

where $\left\langle E_{i}(\mathbf{r}) E_{k}\left(\mathbf{r}^{\prime}\right)\right\rangle_{\omega}$, the spectral correlation tensor, relates to the retarded Green function as

$$
\left\langle E_{i}(\mathbf{r}) E_{k}\left(\mathbf{r}^{\prime}\right)\right\rangle_{\omega}=\operatorname{Im} G_{i k}^{R}\left(\mathbf{r}, \mathbf{r}^{\prime}, \omega\right) \operatorname{coth}\left(\frac{1}{2} \beta \omega\right), \quad \beta=1 /\left(k_{B} T\right)
$$

(this is the fluctuation-dissipation theorem). Unless the Green function has an imaginary part, the two-point function is simply zero. We thus see, also via this alternative route, that absorption is necessary.

Different strategies have been followed in order to deal with the Casimir friction problem. The one followed here will be to adopt a microscopic model, in which we start from the statistical mechanics for harmonically oscillating particles at finite temperature moving nonrelativistically with constant velocity. Such a statisticallymechanical model is of course much more simple than the dielectric model shown in Fig. 1. We argue, however, that such a microscopic model is able to elucidate the essentials of the Casimir friction.

The presentation presented here is based on our three recent papers $11|12| 13$. In turn, they are based on two papers of ours some years ago 14|15. The microscopic model has been analyzed by other investigators also, in particular by Barton recently 16 17/18. We shall have the opportunity to compare with some of his results below.

The other main avenue of approach in order study the friction problem, is to adopt the macroscopic model with permittivity properties. Let us finally refer to some works in that direction: Refs. 19, 20, 21, 22, 23, 24, 25, 


\section{Friction force: Statistical mechanical method}

Consider a quantum mechanical two-oscillator system whose reference state is the one of uncoupled motion corresponding to the Hamiltonian $H_{0}$. The equilibrium situation becomes perturbed by a time dependent term - $A q(t)$, where $A$ is a time independent operator and $q(t)$ a classical function of time whose explicit form depends on the specific properties of the system. The Hamiltonian becomes $H=H_{0}-A q(t)$. We put

$$
\text { - } A q(t)=\psi(\mathbf{r}(t)) x_{1} x_{2},
$$

where $\psi(\mathbf{r})$ is the coupling strength (i.e. $\psi$ is the classical potential between the oscillators). The separation between the oscillators is $\mathbf{r}$, and $x_{1}, x_{2}$ are the internal vibrational coordinates of the oscillators. When the oscillators move with respect to each other the coupling has to vary in time. With nonrelativistic constant relative velocity $\mathbf{v}$ the interaction will vary as

$$
-A q(t)=\left[\psi\left(\mathbf{r}_{0}\right)+\nabla \psi\left(\mathbf{r}_{0}\right) \cdot \mathbf{v} t+\ldots\right] x_{1} x_{2},
$$

when expanded around the initial position $\mathbf{r}=\mathbf{r}_{0}$ at $t=0$. The force between the oscillators, called $\mathbf{B}$, is

$$
\mathbf{B}=-(\nabla \psi(\mathbf{r})) x_{1} x_{2}
$$

Note that in a mathematical sense the expansion (7) requires $v t$ to be small. Physically, we assume nevertheless Eq. (7) to hold for all times, so that the interaction energy is proportional to $t$ for all values of $t$. The natural opportunity of choosing $q(t)=t$ in the interaction (6) thus has to be modified: as will be shown below, a convergence factor will be needed.

Another point worth noticing is that the expression (6) corresponds to first quantization only. Quantum electrodynamic processes such as emission and absorption of photons (second quantization) are not accounted for by the present model. They were considered, however, in Ref. 15 .

The equilibrium situation with both oscillators at rest is represented by the first term in (77). It gives rise to a (reversible) equilibrium force. Thus the friction must be connected with the second term. To simplify, we will for the moment neglect the first term. By this the two oscillators will be fully uncorrelated in their relative position $\mathbf{r}=\mathbf{r}_{0}$. The friction force, due to the time dependence of the interaction (7), will be a small perturbation upon the equilibrium situation. This interaction leads to a response $\Delta\langle\mathbf{B}(t)\rangle$ in the thermal average of $\mathbf{B}$. And this is where the Kubo formula comes in:

$$
\Delta\langle\mathbf{B}(t)\rangle=\int_{-\infty}^{t} \phi_{B A}\left(t-t^{\prime}\right) q\left(t^{\prime}\right) d t^{\prime}
$$

(note that here $\phi_{B A}$ means a vector), where the response function is given by

$$
\phi_{B A}(t)=\frac{1}{i \hbar} \operatorname{Tr}\{\rho[\mathrm{A}, \mathbf{B}(\mathrm{t})]\} .
$$


Here $\rho$ is the density matrix and $\mathbf{B}(t)$ is the Heisenberg operator $\mathbf{B}(t)=$ $e^{i t H / \hbar} \mathbf{B} e^{-i t H / \hbar}$, where $\mathbf{B}$ like $A$ is time independent. Now with (7) and (8), and with $q(t)=t$, expression (10) can be rewritten as $\phi_{B A}(t)=\mathbf{G} \phi(t)$, with

$$
\begin{gathered}
\mathbf{G}=(\nabla \psi)(\mathbf{v} \cdot \nabla \psi), \quad \phi(t)=\operatorname{Tr}\{\rho \mathrm{C}(\mathrm{t})\}, \\
C(t)=\frac{1}{i \hbar}\left[x_{1} x_{2}, x_{1}(t) x_{2}(t)\right] .
\end{gathered}
$$

Thus with Eq. (9) and $q\left(t^{\prime}\right)=t^{\prime}$ the force can be written as

$$
\mathbf{F}=\Delta\langle\mathbf{B}(t)\rangle=\mathbf{G} \int_{-\infty}^{t} \phi\left(t-t^{\prime}\right) t^{\prime} d t^{\prime}=\mathbf{F}_{r}+\mathbf{F}_{f}
$$

where

$$
\mathbf{F}_{r}=\mathbf{G} t \int_{0}^{\infty} \phi(u) d u
$$

is part of the reversible force by which the part of the force that represents friction is

$$
\mathbf{F}_{f}=-\mathbf{G} \int_{0}^{\infty} \phi(u) u d u
$$

Here the new variable $u=t-t^{\prime}$ has been introduced. The $\mathbf{F}_{r}$ can be interpreted as a reversible force since it depends only upon position. This interpretation is consistent with the result obtained for the dissipation below; the $\mathbf{F}_{r}$ will not contribute to the net total dissipation.

If one again includes $\psi\left(\mathbf{r}_{0}\right)$ one has

$$
\mathbf{G} t=(\nabla \psi) \psi\left(\mathbf{r}_{0}+\mathbf{v} t\right),
$$

where $\mathbf{r}=\mathbf{r}_{0}+\mathbf{v} t$ is the position at time $t$. By contrast, expression (13) changes sign when the velocity $\mathbf{v}$ changes sign, and it thus represents a friction force. (Observe that the velocity in (14) merely represents the shift in position.) Equation (13) is the same as result (2.11) in Ref. 14 a, and the Fourier transformed version of it and Eq. (13) above as well, is

$$
\mathbf{F}_{f}=-\left.i \mathbf{G} \frac{\partial \tilde{\phi}(\omega)}{\partial \omega}\right|_{\omega=0}
$$

where $\tilde{\phi}(\omega)=\int_{0}^{\infty} \phi(t) e^{-i \omega t} d t \quad($ with $\phi(t)=0$ for $t<0)$.

In Ref. 14 the Fourier transformed version (15) was used to obtain the explicit expression for the friction force. Here, we instead will use a different approach based on the expression (13). As in the reference mentioned we then need the commutator (11). This entity again follows from the properties of quantized harmonic oscillators.

a There is a missing minus sign in Eq. (2.11) and the $\mathbf{F}_{r}$ was not taken into account. 
We introduce annihilation and creation operators $a$ and $a^{\dagger}$ with commutation relations $\left[a_{i}, a_{i}^{\dagger}\right]=1\left(i=1,2\right.$; other commutators vanish). As usual, $a_{j}(t)=a_{j} e^{-i \omega_{j} t}$ and $a_{j}^{\dagger}(t)=a_{j}^{\dagger} e^{i \omega_{j} t}$. With this the coordinates are

$$
x_{i}=\left(\frac{\hbar}{2 m_{i} \omega_{i}}\right)^{1 / 2}\left(a_{i}+a_{i}^{\dagger}\right)
$$

where $m_{i}$ and $\omega_{i}(i=1,2)$ are the mass and eigenfrequency of each oscillator. To obtain $\phi(t)$ from (11) we first have to calculate $\phi(t)=\left\langle\left\langle n_{1} n_{2}|C(t)| n_{1} n_{2}\right\rangle\right\rangle$, where $\left|n_{1} n_{2}\right\rangle=\left|n_{1}\right\rangle\left|n_{2}\right\rangle$ represents eigenstates with oscillators excited to levels $n_{1}$ and $n_{2}$. Some calculation yields

$$
\phi(t)=D\left[\left(2\left\langle n_{1}\right\rangle+1\right) \cos \left(\omega_{1} t\right) \sin \left(\omega_{2} t\right)+\left(2\left\langle n_{2}\right\rangle+1\right) \cos \left(\omega_{2} t\right) \sin \left(\omega_{1} t\right)\right],
$$

where $D=\hbar /\left(2 m_{1} m_{2} \omega_{1} \omega_{2}\right)$. The energy levels are $\varepsilon_{n}=\left(n+\frac{1}{2}\right) \hbar \omega$.

In Ref. 14 the expression (17) was Fourier transformed to obtain the friction force as given by (15). As an alternative we will here use expression (17) directly in Eq. (13). Then we get the integral 11

$$
\begin{gathered}
\int_{0}^{\infty} t e^{-\eta t} \cos \left(\omega_{1} t\right) \sin \left(\omega_{2} t\right) d t \\
=\frac{\eta \Omega_{1}}{\left(\eta^{2}+\Omega_{1}^{2}\right)^{2}}-\frac{\eta \Omega_{2}}{\left(\eta^{2}+\Omega_{2}^{2}\right)^{2}} \rightarrow-\frac{\pi}{2 \Omega_{2}} \delta\left(\Omega_{2}\right), \quad \eta \rightarrow 0 .
\end{gathered}
$$

Here $\Omega_{1}=\omega_{1}+\omega_{2}$ and $\Omega_{2}=\omega_{1}-\omega_{2}$. As mentioned above a convergence factor $e^{-\eta t}$ is needed, and the limit $\eta \rightarrow 0$ is taken. Then the $\Omega_{2}$-term becomes a delta function with prefactor determined by the integral $\int_{-\infty}^{\infty} \eta x^{2}\left(\eta^{2}+x^{2}\right)^{-2} d x=\pi / 2$. From (17) we also get this integral with $\omega_{1}$ and $\omega_{2}$ interchanged. This will then give the result (18) with opposite sign with respect to the $\Omega_{2}$-term. Adding up we get the difference of the prefactors

$$
\begin{gathered}
\operatorname{coth}\left(\frac{1}{2} \beta \hbar \omega_{1}\right)-\operatorname{coth}\left(\frac{1}{2} \beta \hbar \omega_{2}\right)=-\frac{\sinh \left(\frac{1}{2} \beta \hbar \Omega_{2}\right)}{\sinh \left(\frac{1}{2} \beta \hbar \omega_{1}\right) \sinh \left(\frac{1}{2} \beta \hbar \omega_{2}\right)} \\
\rightarrow-\frac{\frac{1}{2} \beta \hbar \Omega_{2}}{\sinh \left(\frac{1}{2} \beta \hbar \omega_{1}\right) \sinh \left(\frac{1}{2} \beta \hbar \omega_{2}\right)}, \quad \eta \rightarrow 0 .
\end{gathered}
$$

Multiplying (18) with (19) and including the factors $D$ and $\mathbf{G}$ the friction force becomes 11

$$
\mathbf{F}_{f}=-\frac{\pi \beta \hbar^{2}(\nabla \psi)(\mathbf{v} \cdot \nabla \psi)}{8 m_{1} m_{2} \omega_{1}^{2} \sinh ^{2}\left(\frac{1}{2} \beta \hbar \omega_{1}\right)} \delta\left(\omega_{1}-\omega_{2}\right),
$$

which is also the result (3.14) of Ref. 14. Again one notes that there is friction only when the oscillators have the same frequency, and $\beta$ should be finite, i.e. $T>0$.

When $\beta \rightarrow \infty$, the expression (20) vanishes. According to the present oscillator model there is thus no friction force at zero temperature. An objection against this result may be that it is somewhat singular due to the presence of the $\delta$-function. 
Thus its physical significance may not be obvious. However, $\eta$ can be kept finite. This will smooth out the $\delta$-function, and the $\Omega_{1}$-term in Eq. (18) will give a contribution too. Note that this will not change our conclusions about a finite friction force for $T>0$. (For finite $\eta$, i.e. interaction like a short pulse, there will also be a contribution for $T=0$ due to the $\Omega_{1}$-term in Eq. (18).) But here we will assume $\eta$ small by which the $T=0$ contribution can be disregarded.

In Ref. 14 the result (20) for the friction force was derived also by two other methods. These methods utilized the path integral formalism of quantum systems at thermal equilibrium 26. The path integral can be identified with a classical polymer problem where imaginary time is a fourth dimension of length $\beta$. Thus the polymers stretch out in the fourth dimension and form closed loops of periodicity $\beta$. For harmonic oscillators the correlation function along the polymers is obtained in a straightforward way. With one of the methods the convolution of the correlation functions of both oscillators were needed. The resulting Fourier transform of this convolution was then identified with the response function $\tilde{\phi}(\omega)$ used in the expression (15) 27.

With the other method full thermal equilibrium was utilized. Then the relative motion of the oscillators was regarded as a harmonic oscillator motion with low frequency $\omega_{0} \rightarrow 0$. Again with the path integral one can obtain the Fourier transform of the response function for the relative motion. The damping of the relative motion, that can be related to this response function, gives the friction force, and again the result (20) was recovered. Thus the three methods used in Ref. 14, as well as the modification considered in the present paper, all lead to the same result, in contradiction to some other results in the literature, for instance that of Ref. 25.

The result for the friction force was also extended to the situation with timedependent or non-instantaneous interaction 15 . Then the full thermal equilibrium method was applicable to generalize the result. With the latter interaction there was also a friction from the self-interaction of a moving oscillator with itself.

Consider now the dissipation of energy. It is identified with the work done, during a finite time interval starting from $t=0$ with maximum velocity $\mathbf{v}$ when the position is $\mathbf{r}=\mathbf{r}_{0}$. As $t \rightarrow \infty$ the motion is required to die out. To accomplish this we again introduce the convergence factor $e^{-\eta t}(\eta \rightarrow 0)$, whereby $t$ is to be replaced with $q(t)=t e^{-\eta t}$. The velocity decays exponentially,

$$
\mathbf{v} \rightarrow \mathbf{v}_{1}(t)=\mathbf{v} \dot{q}(t)=\mathbf{v}(1-\eta t) e^{-\eta t}
$$

For $\eta t>0, \mathbf{v}_{1}(t)$ will now replace $\mathbf{v}$ in expression (20) for the friction force. Altogether, the total energy dissipated will be

$$
\Delta E=-\int_{-\infty}^{\infty} \mathbf{v}_{1}(t) \cdot \mathbf{F}_{f} \dot{q}(t) d t=-\mathbf{v} \cdot \mathbf{F}_{f} \int_{0}^{\infty}[\dot{q}(t)]^{2} d t=-\frac{1}{4 \eta} \mathbf{v} \cdot \mathbf{F}_{f},
$$

where $\mathbf{F}_{f}$ is given by Eq. (20) Dote that the reversible part of the force $\mathbf{F}_{r} \propto t \rightarrow$

${ }^{\mathrm{b}}$ The minus signs are missing in Eq. (21) of Ref. 11 
$q(t)$ as given by Eq. (13) will not contribute to the dissipation since $\int_{0}^{\infty} \dot{q}(t) q(t) d t=$ 0 .

\section{Energy dissipation calculated from first order perturbation theory}

Our intention now is to calculate the change $\Delta E$ in energy by means of quantum mechanical perturbation theory; this was considered also in Ref. 12, It turns out that the change in energy occurs to second order in the perturbation. Nevertheless, time-dependent perturbation theory to the first order is sufficient to find this second order effect. This is because the phases of the perturbed change in amplitudes, and the initial amplitudes of the eigenstates, are uncorrelated at thermal equilibrium. Thus change in amplitudes of eigenstates will be the square of perturbed amplitudes; i.e. there are no cross-terms. We find that $\Delta E$ is positive, corresponding to a friction force. Doubts occasionally raised in the literature about the very existence of the Casimir friction effect 25 are thus from this standpoint laid at rest.

Making use of the expression for $\Delta E$ we compare the formalism of the present section with that of Ref. 11, and Sec. 2 above. There, the linear response via the Kubo formalism was used $\frac{14}{15}$ to calculate the force which in turn could be divided into a reversible and an irreversible part. It is the latter part that is associated with dissipation. A satisfactory feature is that the derivation in the present section, although being quite different from that of Ref.11, leads to the same physical result.

To fix the notation, we start with perturbation theory for a system at thermal equilibrium. The wave function can be written as $\psi=\sum_{n} a_{n} \psi_{n}$, where $\psi_{n}=\psi_{n}(x)$ are the eigenstates. For simplicity we here let $x$ represent all the coordinates of the system. If $\psi$ is normalized, $\int \psi^{*} \psi d x=1$, then $\left|a_{n}\right|^{2}$ is the probability for the system to be in eigenstate $n$. At thermal equilibrium this probability is given by the Boltzmann factor $P_{n}=\left|a_{n}\right|^{2}=Z^{-1} e^{-\beta E_{n}}$, where $E_{n}$ is the energy eigenvalue of the state and $Z$ is the partition function $Z=\sum_{n} e^{-\beta E_{n}}$. Let now the Hamiltonian be perturbed by the time-dependent interaction $V(t)=-A q(t)$, where $A$ is a quantum mechanical operator while $q(t)$ is a scalar function. The $A$ is time independent.

Due to the perturbation the coefficients $a_{n}$ will change. If the system starts in a state $m$ there are transitions to other states given by a change in $a_{n}, \Delta a_{n}=b_{n m}$. The $b_{n m}$ is given by the standard expression

$$
b_{n m}=\frac{1}{i \hbar} \int_{-\infty}^{t} V_{n m}(\tau) e^{i \omega_{n m} \tau} d \tau,
$$

where

$$
V_{n m}(\tau)=\int \psi_{n}^{*} V(\tau) \psi_{m} d x=-A_{n m} q(\tau)
$$

$A_{n m}=\langle n|A| m\rangle$. Here $\omega_{n m}=\omega_{n}-\omega_{m}$, with $\omega_{n}=E_{n} / \hbar$.

As mentioned above, we will assume that the perturbation vanishes after some 
time. Then we will obtain the total change in $\Delta a_{n}$ with

$$
b_{n m}=-\frac{1}{i \hbar} A_{n m} \hat{q}\left(-\omega_{n m}\right), \quad \hat{q}(\omega)=\int_{-\infty}^{\infty} q(t) e^{-i \omega t} d t,
$$

where the hat denotes Fourier transform.

From a general perspective, the system may start in a combination of eigenstates with transitions from several states. With this, $\Delta a_{n} \rightarrow \sum_{m \neq n} a_{m} b_{n m}$. Now, the state $n$ does not only receive contributions, but gives away contributions to other states also. The latter must follow from the corresponding increase of probabilities for the other states. Omitting the latter for the moment, the perturbed coefficients are

$$
a_{1 n}=a_{n}+\Delta a_{n}=a_{n}+\sum_{m \neq n} a_{m} b_{n m} .
$$

The $a_{n}$ will have complex phase factors, and in thermal equilibrium one must assume the phases of $a_{n}$ and $a_{m}(m \neq n)$ to be uncorrelated. Thus by thermal average, $\left\langle a_{n}^{*} a_{m}\right\rangle=0$. With this the new probability of the state $n$ becomes

$$
P_{1 n}=\left\langle a_{1 n}^{*} a_{1 n}\right\rangle=\left|a_{n}\right|^{2}+\sum_{m \neq n}\left|a_{m}\right|^{2} B_{n m},
$$

where $B_{n m}=b_{n m} b_{n m}^{*}=\left|b_{n m}\right|^{2}$. The last term is the increase in probability from the other states. Likewise, the state $n$ must obey a similar loss of probability to other states to conserve probability. The loss to other states is thus $\sum_{m \neq n}\left|a_{n}\right|^{2} B_{m n}$. With Eq. (23) we have $b_{m n}=b_{n m}^{*}$, by which $B_{m n}=B_{n m}$. The latter equation reflects that the transition probabilities between each pair of states are the same in either direction. With this, the resulting perturbed probability of state $n$ becomes

$$
P_{1 n}=P_{n}+\sum_{m}\left(P_{m}-P_{n}\right) B_{n m} .
$$

The change in energy can now be evaluated as

$$
\Delta E=\sum_{n m} E_{n}\left(P_{m}-P_{n}\right) B_{n m}=\sum_{n m}\left(E_{n}-E_{m}\right) P_{m} B_{n m} .
$$

Utilizing the symmetry with respect to $n$ and $m$ we find

$$
\Delta E=\frac{1}{Z} \sum_{n m} e^{-\frac{1}{2} \beta\left(E_{n}+E_{m}\right)} \Delta_{n m} \sinh \left(\frac{1}{2} \beta \Delta_{n m}\right) B_{n m},
$$

with $\Delta_{n m}=E_{n}-E_{m}$, and where

$$
B_{n m}=\left(1 / \hbar^{2}\right) A_{n m} A_{n m}^{*} \hat{q}\left(-\omega_{n m}\right) \hat{q}\left(\omega_{n m}\right) .
$$

Note that $\Delta E \geq 0$. The dissipation occurs to second order in the perturbation. To first order there is no dissipation; the changes are adiabatic. 


\section{Energy dissipation from friction force}

According to the statistical mechanical approach above - Sec. 2 and Ref. 11- the energy dissipation can be written in the form

$$
\Delta E=-\int_{-\infty}^{\infty} v(t) F_{f} d t=-\int_{-\infty}^{\infty}\left[\int_{-\infty}^{t} \dot{q}(t) \phi_{A A}\left(t-t^{\prime}\right) q\left(t^{\prime}\right) d t^{\prime}\right] d t
$$

cf. Eq. (27) in Ref. 11 (the minus sign in front of the integrals is missing in that reference). The quantity $q(t)$ is most naturally connected with the position, $x(t)=$ $q(t)$, but it can also be interpreted as a position in a more generalized sense as discussed in Refs. 11 and 12, so that the result (30) has a broader applicability. We will now show that this is actually the case, by showing that the result (30) is the same as (28), obtained by means of time-dependent perturbation theory.

With wave function representation we first have

$$
\begin{gathered}
e^{-\beta H} \rightarrow \sum_{n} \psi_{n}(x) e^{-\beta E_{n}} \psi_{n}^{*}\left(x_{1}\right), \\
\rho A A(t)=\frac{1}{Z} \sum_{n m k} \int \psi_{n}(x) e^{-\beta E_{n}} \psi_{n}^{*}\left(x_{1}\right) A \psi_{m}\left(x_{1}\right) e^{i \omega_{m} t} \psi_{m}^{*}\left(x_{2}\right) A \\
\times \psi_{k}\left(x_{2}\right) e^{-i \omega_{k} t} \psi_{k}^{*}\left(x_{3}\right) d x_{1} d x_{2} .
\end{gathered}
$$

Thus we obtain

$$
\operatorname{Tr}(\rho A A(t))=\frac{1}{Z} \sum_{n m} e^{-\beta E_{n}} A_{n m} e^{i \omega_{m} t} A_{m n} e^{-i \omega_{n} t},
$$

as $\int \psi_{k}^{*}(x) \psi_{n}(x) d x=\delta_{k n}\left(x_{3}=x_{1}=x\right)$, and $A_{n m}=\langle n|A| m\rangle$. Likewise we calculate $\operatorname{Tr}(\rho A(t) A)$ by exchange of $\omega_{n}$ and $\omega_{m}$ in Eq. (33). The response function becomes

$$
\phi_{A A}(t)=\frac{1}{i \hbar} \operatorname{Tr}\{\rho[A, A(t)]\}=\frac{1}{i \hbar} \sum_{n m} M_{n m}\left(e^{-i \omega_{n m} t}-e^{i \omega_{n m} t}\right),
$$

with

$$
M_{n m}=-\frac{1}{Z} e^{-\frac{1}{2} \beta\left(E_{n}+E_{m}\right)} \sinh \left(\frac{1}{2} \beta \Delta_{n m}\right) A_{n m} A_{n m}^{*}
$$

(recall that $\Delta_{n m}=E_{n}-E_{m}=\hbar \omega_{n m}, A_{m n}=A_{n m}^{*}$ ). The expression for $M_{n m}$ follows if one first exchanges $n$ and $m$ in Eq. (33), then adds the resulting term to it and divides by 2 . Some manipulation then yields

$$
\Delta E=\frac{1}{\hbar} \sum_{n m} M_{n m} \omega \hat{q}(\omega) \hat{q}(-\omega) .
$$

With $\omega=\omega_{n m}=\Delta_{n m} / \hbar$ and $M_{n m}$ given by the expression (35) this is nothing but the result (28), but now obtained by time-dependent perturbation theory. Thus we have been able to derive the same expression for the dissipated energy in two independent ways. 
The result (28) (or (36)) may be applied to the pair of interacting harmonic oscillators considered in Sec. 2, but we abstain from further details here; the reader is referred to Ref. 12, We give, however, the final expression for $\Delta E$ :

$$
\Delta E=\frac{\pi \beta \hbar^{2} \gamma^{2}}{8 \eta \sinh ^{2}\left(\frac{1}{2} \beta \omega_{1}\right)} \delta\left(\omega_{1}-\omega_{2}\right)
$$

where

$$
\gamma=\left(\frac{1}{2} D \hbar\right)^{1 / 2}(\mathbf{v} \cdot \nabla \psi)
$$

with $D$ given below Eq. (17). This is the same as the result (22), with expression (20) inserted.

We may summarize our developments of this section:

1. The total energy dissipation was calculated for a system perturbed by a varying interaction. The change in energy is basically a second order effect but was calculated with the use of standard time-dependent perturbation theory to first order only, the reason being the absence of cross terms due to uncorrelated phases of eigenstates. The energy change was found to be positive or zero. The result agrees with our previous results of Ref. 11, obtained in a different and independent way.

2. We have in general assumed finite temperature, and initial thermal equilibrium. Moreover, we have assumed low velocities and nonrelativistic mechanics, whereby photons are not present. Photons were introduced, however, in our earlier study 15 .

3. The energy change $\Delta E$ is finite in general. This corresponds to a finite friction force. In the limit $T \rightarrow 0$ our formalism gives, however, that $\Delta E \rightarrow 0$ for the model considered in Sec. 2. This result is due to our assumption about constant velocity, involving slowly varying coupling. For couplings varying more rapidly, there will also be a friction force at $T=0$, due to transitions to excited states.

\section{Equivalence between different formulations}

As mentioned, there are different formulations in the literature on how to deal with Casimir friction. In the present section, assuming $T=0$, we wish to compare our formalism above with that recently given by Barton 16$] 17$ (cf. also Ref. 18 dealing with finite temperature). In particular, Ref. 16 dealt with a two-oscillator model, thus in essence the same microscopic model as ours. Barton analyzed the system using quantum mechanical perturbation theory. The striking point is that the expressions he obtained are seemingly quite different from those we obtained in Ref. 11, In Barton's own words (Ref. 16, Sect. 3) "..in view of the manifold current controversies about quantum-governed frictional force generally, it seems well worth exploring whether such differences reflect substantiate disagreement or only a confusion of terms". Our present investigation is a follow-up of Barton's suggestion. We intend to demonstrate explicitly that the obtained expressions for 
the dissipated Casimir energy are in fact in agreement with each other, thus a reassuring result.

Assume that the oscillators have the same eigenfrequency $\omega$ and the same mass $m$. They interact via a time-dependent Hamiltonian

$$
H_{\mathrm{int}}=\frac{e^{2}}{s^{3}} y_{1} y_{2}
$$

(Gaussian units assumed). Here $e$ is the elementary charge, $y_{1}$ and $y_{2}$ the oscillator coordinates, and $\mathbf{s}=\mathbf{s}(t)$ is the vectorial distance between the mass centers, varying with time because of the relative motion of the oscillators. Introducing new coordinates

$$
y_{ \pm}=\frac{y_{1} \pm y_{2}}{\sqrt{2}}
$$

one can write the interaction Hamiltonian as

$$
\begin{gathered}
H_{\mathrm{int} \pm}=H_{\mathrm{int}+}+H_{\mathrm{int}-}, \\
H_{\mathrm{int} \pm}= \pm \frac{1}{2} q y_{ \pm}^{2}, \quad q=\frac{e^{2}}{s^{3}} .
\end{gathered}
$$

By use of time-dependent perturbation theory the total energy dissipated is then found to be

$$
\begin{gathered}
\Delta E=2 \times 2 \hbar \omega|c(\infty)|^{2} \\
c(t)=-\frac{i}{2 \hbar} \int_{-\infty}^{t} d t^{\prime} q\left\langle 2_{+}\left|y_{+}^{2}\right| 0_{+}\right\rangle e^{2 i \omega t^{\prime}},
\end{gathered}
$$

as given by Eqs. (3.3) and (2.4) respectively, in Ref. 16. At $T=0$, only excitations from the ground state are possible.

To compare Barton's result (42) with ours, we first have to evaluate the matrix elements in (42). In terms of the creation and annihilation operators we have $\left(\omega_{ \pm} \rightarrow\right.$ $\omega$ for small perturbations)

$$
y_{ \pm}=\sqrt{b}\left(a_{ \pm}+a_{ \pm}^{\dagger}\right), \quad b=\frac{\hbar}{2 m \omega} .
$$

Then,

$$
\left\langle 2_{+}\left|y_{+}^{2}\right| 0_{+}\right\rangle=b\left\langle 2_{+}\left|a_{+}^{\dagger}{ }^{2}\right| 0_{+}\right\rangle=\sqrt{2} b .
$$

Together with Eq. (42) this gives

$$
\begin{gathered}
\Delta E=8 \hbar \omega b^{2}|I(\infty)|^{2} \\
I(t)=-\frac{i}{2 \hbar} \int_{-\infty}^{t} d t^{\prime} q e^{2 i \omega t^{\prime}} .
\end{gathered}
$$


Proceed now to compare this result with those that we derived in Refs. 11 and 12 , at $T=0$. The interaction Hamiltonian, written as $H_{\mathrm{int}}=-A q(t)$, now corresponds to

$$
A=-y_{1} y_{2}, \quad \text { and } \quad q(t)=q=\frac{e^{2}}{s^{3}} .
$$

Recall that $A$ is a time-independent operator accounting for the quantum mechanical properties of the system, while $q(t)$ is a classical function of time. At $T=0$ the system is in its ground state with probability equal to one.

The change in energy is given by Eq. (27), where $E_{n}$ is the energy in the (unperturbed) eigenstate $n$, and $P_{n}=\left|a_{n}\right|^{2}$ is the probability of the system to be in this state. As mentioned, we start from the ground state so that $P_{m} \rightarrow P_{00}=1$. Further, as $B_{n m}=\left|b_{n m}\right|^{2}$ with $b_{n m}$ the transition coefficient between states $m$ and $n$, Eq. (27) reduces to

$$
\Delta E=\left(E_{11}-E_{00}\right) B_{1100},
$$

where $E_{11}-E_{00}=2 \hbar \omega$ is the energy difference between the state $|11\rangle$ where both oscillators are excited to the first level, and the ground state $|00\rangle$. The coefficient $B_{1100}$ is the transition probability between these two states.

What remains is to calculate $B_{1100}$. Using Eq. (24) we get

$$
\hat{q}\left(-\omega_{n m}\right) \rightarrow \hat{q}\left(-\omega_{1100}\right)=\hat{q}(-2 \omega)=2 i \hbar I(\infty),
$$

with $I(\infty)$ given by Eq. (11). Further,

$$
\begin{gathered}
A_{n m} \rightarrow A_{1100}=\left\langle 11\left|-y_{1} y_{2}\right| 00\right\rangle \\
=-\left\langle 1\left|y_{1}\right| 0\right\rangle\left\langle 1\left|y_{2}\right| 0\right\rangle=-\left\langle 1\left|y_{1}\right| 0\right\rangle^{2}=-b\left\langle 1\left|a_{1}^{\dagger}\right| 0\right\rangle^{2}=-b .
\end{gathered}
$$

Altogether, when inserted into Eq. (47) we obtain

$$
\begin{gathered}
B_{1100}=\frac{1}{\hbar^{2}}\left|A_{1100}\right|^{2} \hat{q}(-2 \omega) \hat{q}(2 \omega)=4 b^{2}|I(\infty)|^{2}, \\
\Delta E=8 \hbar \omega b^{2}|I(\infty)|^{2},
\end{gathered}
$$

which coincides with the result obtained by Barton, Eq. (42) above.

As an additional remark we note that the situation with zero friction at $T=0$ for slowly varying forces can be analyzed in a straightforward way from the equations above. With time-dependent part $(q=0, t<0)$,

$$
q(t)=t e^{-\eta t}, \quad t>0
$$

we namely find when $\eta \rightarrow 0$

$$
\begin{aligned}
\hat{q}(\omega) & =\frac{1}{(\eta+i \omega)^{2}}, \\
\hat{q}(\omega) \hat{q}(-\omega) & =\frac{1}{\left(\eta^{2}+\omega^{2}\right)^{2}} \rightarrow \frac{\pi}{2 \eta \omega^{2}} \delta(\omega) .
\end{aligned}
$$


Thus with $\omega \neq 0$, Eq. (53) will give zero for the dissipated energy $\Delta E$.

[By looking at this in some more detail it would seem that the middle term in Eq. (53), before taking the limit $\eta \rightarrow 0$, implies there to be some dissipation. This is physically an artefact, due to our assumption of an abrupt change of $q(t)$ at $t=0$.]

The equivalence between Barton's results and ours is therewith shown. We thus hope to have shed light on one of the subtle issues in the Casimir friction world demonstrating that quite different approaches can lead to the same result.

\section{Acknowledgment}

I.B. thanks Gabriel Barton for valuable correspondence.

\section{References}

1. A. Ashkin and J. M. Dziedzic, Phys. Rev. Lett. 30, 139 (1973).

2. H. M. Lai and K. Yang, Phys. Rev. A 14, 2329 (1976).

3. I. Brevik, Phys. Reports 52, 133 (1979).

4. J.-Z. Zhang and R. K. Chang, Opt. Lett. 13, 916 (1988).

5. H. M. Lai et al., J. Opt. Soc. Am. B 6, 2430 (1989).

6. I. Brevik and R. Kluge, J. Opt. Soc. Am. B 16, 976 (1999).

7. A. Casner and J.-P. Delville, EPL 65, 337 (2004).

8. J.-P. Delville et al., J. Opt. A: Pure Appl. Opt. 11, 034015 (2009).

9. R. Wunenburger et al., J. Fluid Mech. 666, 273 (2011).

10. J. Schwinger, L. L. DeRaad, Jr. and K. A. Milton, Ann. Phys. 115, 1 (1978).

11. J. S. Høye and I. Brevik, EPL 91, 60003 (2010).

12. J. S. Høye and I. Brevik, Eur. Phys. J. D 61, 335 (2011).

13. J. S. Høye and I. Brevik, Eur. Phys. J. D 64, 1 (2011).

14. J. S. Høye and I. Brevik, Physica A 181, 413 (1992).

15. J. S. Høye and I. Brevik, Physica A 196, 241 (1993).

16. G. Barton, New J. Phys. 12, 113044 (2010).

17. G. Barton, New J. Phys. 12, 113045 (2010).

18. G. Barton, New J. Phys. 13, 043023 (2011).

19. L. S. Levitov, Europhys. Lett. 8, 499 (1989).

20. J. B. Pendry, J. Phys.: Condens. Matter 9, 10301 (1997).

21. J. B. Pendry, New J. Phys. 12, 033028 (2010).

22. A. I. Volokitin and B. N. J. Persson, Phys. Rev. B 78, 155437 (2008).

23. G. V. Dedkov and A. A. Kyasov, J. Phys.: Condens. Matter 20, 354006 (2008).

24. G. V. Dedkov and A. A. Kyasov, Surface Sci. 604, 562 (2010).

25. T. G. Philbin and U. Leonhardt, New J. Phys. 11, 033035 (2009).

26. J. S. Høye and G. Stell, J. Chem. Phys. 75, 5133 (1981).

27. I. Brevik and J. S. Høye, Physica A 153, 420 (1988). 